\title{
THEORY OF THE TOP.
}

Advisory Committee for Aeronautics. Reports and Memoranda, No. 146 . Report on Gyroscopic Theory. By Sir G. GreenHILL. Darling and Son, and T. Fisher Unwin, London, 1914. Folio. iiit 277 pp. +49 figs. Boards. Price 10s.

The British Advisory Committee for Aeronautics has printed, or rather, until the war, had printed annual reports which are the prime sources for information regarding the development of the theory and practice of aeroplane design. Unfortunately they no longer divulge their findings. The Committee has also published other reports bearing less directly upon aeronautical problems, but yet advantageous in the more recondite parts of the theory. Of these supplementary publications, Greenhill's report on gyroscopic theory deserves particular attention of the student of mathematics and mechanics, and cannot forever be neglected by the practical engineer, so important are many instances of gyroscopic action becoming.

We are indeed fortunate that the Committee got Greenhill to prepare the report. His long-continued investigations on the top have made him a world-recognized authority on the subject. The fact that we have available for study the great work of Klein and Sommerfeld on the same subject does not in the slightest detract from the value of the present work. The merest glance will convince any reader that the two treatises are conceived in very different ways and that they rather supplement than overlap one another. Besides Greenhill's is decidedly shorter and more concerned with apparatus. It will be of more interest to the student of mechanics and engineering, though of less to the student of the theory of functions of a complex variable. And here we might remark that a beautiful theory and a practical analysis susceptible to ready calculation are unfortunately not always to be combined. The theta function is a thing of beauty, but I have always found the solution of the problem of the motion of the top by theta functions with complex arguments anything but satisfactory from the point of view of calculation. Perhaps after all Legendre knew what he was about when he introduced his third elliptic integral,- - at any rate we judge that Greenhill thinks so. 
There is a good deal of Greenhillian elliptic analysis in the report, and this may discourage a great many persons; but I desire particularly to emphasize the fact that there is also a great deal of the text which is practically free from elliptic integrals and functions, and which is almost arithmetic and algebraic in its simplicity and directness. The first chapter is on steady gyroscopic motion. Here we have 27 pages that anybody can read. This discussion abounds in the geometric and mechanical illustration for which the author is known. The distinction between strong and weak tops is brought out. The effect of the moving rotating parts of the Gnome engine upon the steering of an aeroplane is shown, also the similar phenomenon in the automobile. Other problems considered are: precession of the equinox, Serson-Fleurias gyroscopic horizon, how to roll a hoop, gyroscopic effect in wheeled carriages and in paddle or screw steamers, stability of an elongated body in a medium (airship or projectile or submarine boat). There is an appendix on general dynamical theory. Naturally, if one is to make this work simple he must concentrate his attention on the vector angular momentum; there is no other way, and the use of this vector is as easy as it is important. Greenhill's first chapter would be valuable for its emphasis on the angular momentum vector even if it contained nothing else of value.

The second chapter is on gyroscopic applications. The simple style of the first chapter is continued. We find treated: the Schlick sea gyroscope, the monorail carriage, the Bessemer saloon, with some mention of steering torpedoes. There is also a section on the theory of oscillation, free, damped, and forced, and an appendix on dynamical units. In both these chapters, as throughout the book, numerous references to the literature are given - to all sorts of literature, not merely to the mathematical.

Chapter III is on the general unsteady motion of the top. The integration rapidly leads to elliptic analysis. The author gives a summary of many ways of treating elliptic integrals and functions. By the mathematician this chapter may be added to the existing tables of formulas. There are, however, other points of interest: the discussion of nearly steady motion, Kirchoff's kinetic analogue of the twisted elastica, the whirling chain, and Clifford's vector treatment of the top. The fourth chapter is largely analytical geometry-Darboux's deformable 
hyperboloid, Poinsot motion, curvature and inflexion of the herpolhode, curvature of the polhode, and the like. Chapters $\mathrm{V}$ and VI are respectively given to the algebraic cases of top motion, and to numerical illustrations and diagrams. This is the ellipsotomic problem. The cases discussed are: bisection of a period, trisection, quinquesection, seven, nine, and eleven section. Various improvements on the author's work in the Annals of Mathematics are given. The analysis will interest the mathematician, the figures will illustrate to anybody a number of cases of motion of the top. The applications are: nutation of the earth's axis, free Eulerian precession and variation of latitude, lunar nutation, and gyroscopic motion of the moon's node. In Chapter VII under the caption, the spherical pendulum, the author treats steady motion and small nutation, and the plane revolving catenary.

With the eighth chapter we come to motion referred to moving origin and axes, and the problems of the motion of a pendulum body and gyroscope about an axle on a whirling arm, of the sphere containing a flywheel and rolling on a table, of the modification of Poinsot motion when the body contains a flywheel spinning on an axle fixed in the body, of the expanding or contracting body, of Kelvin's liquid gyrostat, and finally of the motion of a solid, perforated or not, in an infinite liquid. A knowledge of elliptic functions is not particularly important for the reader of this chapter. In the following ninth and closing chapter Greenhill takes up dynamical problems of steady motion and small oscillations: general motion of a body rolling on a table with attention to steady motion and small oscillations, motion of a body sliding on a smooth table, the single track gyroscopic car, the gyrocompass, Gilbert's barogyroscope, the gyrostatic chain, Kelvin's flexible wire suspension, the gyroscopic pendulum with wire suspension, the stability of a revolving shaft, and the motion of a body with altazimuth suspension. In many of these problems elliptic functions are not mentioned.

It will be seen that the text divides itself naturally into two major parts. Chapters I, II, VIII, and IX deal with practical physical and engineering problems in a practical and on the whole in an elementary way without the use of elliptic functions. There are some sections of the other chapters, III to VII inclusive, which are also elementary, but as a whole these chapters are valuable chiefly to a reader who is not afraid of 
detailed elliptic analysis. This twofold nature of the work is desirable, especially when the division is as clean cut as here, because each type of reader can find and use the part of the book he most needs and at the same time he can gain a glimpse, if not an appreciation, of the sort of work on the same problem which interests the other type of reader.

The forty-nine carefully executed figures are collocated upon large plates at the end of the book. For some of the illustrations this arrangement is satisfactory, but others of them should have been placed at the appropriate places in the text for the readers' ease in following demonstrations. The typography of the work seems excellent. We have now in English a great reference book on the top, a worthy companion and counterpart to that of Klein and Sommerfeld in German, one decidedly more compact and cheaper in price.

Edwin Bidwell WiLson.

Massachusetts Institute of Technology.

\section{NOTES.}

THE second annual meeting of the Mathematical Association of America was held at Columbia University, Thursday to Saturday, December 28-30, 1916. Thursday afternoon was devoted to a joint session with the American Mathematical Society, the American Astronomical Society, and Section A of the American Association for the Advancement of Science, and Thursday evening to a joint dinner of the four organizations. The programme of the Association included addresses by Florian CAJORI: "Discussion of fluxions from Berkeley to Woodhouse"; M. W. Haskell: "University courses in mathematics intended for teachers of secondary mathematics"; T. H. Gronwall: "A nucleus for a mathematical library"; E. B. Wilson: "The mathematics of aerodynamics." On Friday there was an exhibition of portraits and medals of mathematicians from the collection of David Eugene Smith, the presentation and discussion of the report of the library committee, and a business meeting including the election of the following officers: President: Florian Cajori; vicepresidents: D. N. Lehmer and Oswald Veblen; secretary- 